\title{
Molecular Docking Study of Some Novel Combination of Tetrahydroimidazo[1,2-a]pyridine and Trizole Heterocyclics for Anti Staphylococcus Aureus
}

\author{
K. MURALI KRISHNA ${ }^{1}$, P. LEKHA ${ }^{2}$, P K. RAMAN ${ }^{1}$ and P. RAVEENDRA REDDY ${ }^{1 *}$ \\ ${ }^{1}$ Department of Chemistry, Sri Krishna Devaraya University, Anantapuramu-515003, India \\ ${ }^{2}$ SVU College of Engineering, Sri Venkateswara University, Tirupati-517502, India \\ murli39@gmail.com
}

Received 8 March 2016/ Accepted 19 March 2016

\begin{abstract}
Staphylococcus aureus is the most common cause of staph infections such as pneumonia, meningitis, osteomyelitis endocarditis, toxic shock syndrome (TSS) and septicemia. Sortase is an important protein involves in the membrane formation and shape determination in Staphylococcus aureus. We have measured the sortase content in control, Carrier control and experimental groups and found reduction in experimental groups. Using the 3-D structure of Sortase (PDB ID: 4O8L) from Staphylococcus aureus as target we evaluated the binding efficacy of inhibitors with MVD software. The inhibitor binding positions and affinities were determined using MVD scoring fitness functions. With this model, a flexible docking study of Sortase enzyme with derivatives was performed which showed best docking result. Our results may be helpful for further experimental investigations.
\end{abstract}

Keywords: Sortase, Docking, Staphylococcus, MVD software

\section{Introduction}

Sortase enzyme catalyzes transpeptidation reactions on the bacterial surface, utilizing protein precursors with $C$-terminal sorting signals as substrates ${ }^{1}$. Staphylococcus aureus sortase A (SrtA), the prototypic transpeptidase of this class of enzyme ${ }^{2,3}$, cleaves LPXTG motif-type sorting signals between the threonine $(\mathrm{T})$ and the glycine $(\mathrm{G})$ residues to generate an acyl enzyme intermediate ${ }^{4}$. Nucleophilic attack of the amino group of cell wall crossbridges resolves the acyl enzyme, forming an amide bond between the carboxyl group of the $C$-terminal threonine of surface proteins and the cell wall crossbridge of lipid II precursor molecules. The product of this reaction, surface protein linked to lipid II, is then incorporated into the cell wall envelope via the transpeptidation and transglycosylation reactions of peptidoglycan biosynthesis ${ }^{5}$. Twenty different surface proteins with LPXTG motif-type sorting signals have been identified in the staphylococcal genome sequence and deletion of the srtA gene abolishes the cell wall anchoring and surface display of all sortase A substrates. 
As a result, staphylococcal srtA mutants display significant defects in the pathogenesis of murine organ abscesses, infectious arthritis, or endocarditis. The genomes of most gram-positive bacteria encode two or more sortase enzymes, which fulfill different functions. For example, $S$. aureus sortase B is involved in anchoring IsdC (iron-regulated surface determinant C), a polypeptide with an NPQTN motif sorting signal, to the cell wall envelope ${ }^{6}$. Streptococcus pyogenes sortases A and B both anchor surface proteins with LPXTG motif sequences to the envelope. These two sortases appear to recognize unique surface protein substrates using the LPXTG motif as well as other features of cell wall sorting signals. Streptococcal SrtC2 recognizes surface protein substrates with QVPTGV motif sorting signals, consistent with the view that different sortases recognize unique sets of substrates. Perhaps the most astonishing sortase-catalyzed reaction is the assembly of pili on the surface of corynebacteria, actinomycetales, enterococci, group B streptococci and pneumococci. For example, corynebacterial sortases cleave precursor proteins in a manner that leads to the assembly of pili, high-molecular-weight polymerization products several microns long on the bacterial surface. Two domains of pilus surface proteins, the sorting signal and the pilin motif, are required for this reaction, which occurs in a sortase-specific manner. This results in the assembly of different types of pili by dedicated pairs of sortase enzymes and pilin subunit proteins.

\section{Experimental}

\section{Active site identification}

Active site of sortase was identified using CASTp server. A new program, CASTp, for automatically locating and measuring protein pockets and cavities, is based on precise computational geometry methods, including alpha shape and discrete flow theory. CASTp identifies and measures pockets and pocket mouth openings, as well as cavities. The program specifies the atoms lining pockets, pocket openings and buried cavities; the volume and area of pockets and cavities; and the area and circumference of mouth openings.

\section{Docking method}

MVD (Molgrow Virtual Docker) a genetic algorithm based software, mainly utilizes an evolutionary strategy involving 3 genetic operators; cross overs, mutations and migrations. MVD imports the partial flexibility to proteins and full flexibility to inhibitors. The compounds are docked into the active site of Sortase and the interaction of these ligands with the active site residues are thoroughly studied using calculations of molecular mechanics (Figure 1).

The parameters used for GA were population size (100), selection pressure (1.1), number of operations $(10,000)$, number of island (1) and niche size. Operator parameters for crossover, mutation and migration were set to 100,100 and 10 respectively. Default cutoff values of $3.0 \mathrm{~A}^{\circ}(\mathrm{dH}-\mathrm{X})$ for hydrogen bonds and $6.0 \mathrm{~A}^{\circ}$ for vanderswaals were employed. The default algorithm speed was selected and the inhibitor binding site in Sortase was defined within a $10 \mathrm{~A}^{\circ}$ radius with the centroid as HH atom of TYR51, HIS13 and GLN8 respectively. The number of poses for each inhibitor was set 100 and early termination was allowed if the top three bound conformations of inhibitors were within $1.5 \mathrm{~A}^{\circ} \mathrm{RMSD}$. After docking, the individual binding poses of each inhibitor were observed and their interactions with the protein were studied. The best and most energetically favorable conformation of each inhibitor was selected.

\section{Results and Discussion}

Final refined model of sortase domain using SPDBV program and was shown in Figure 2. 

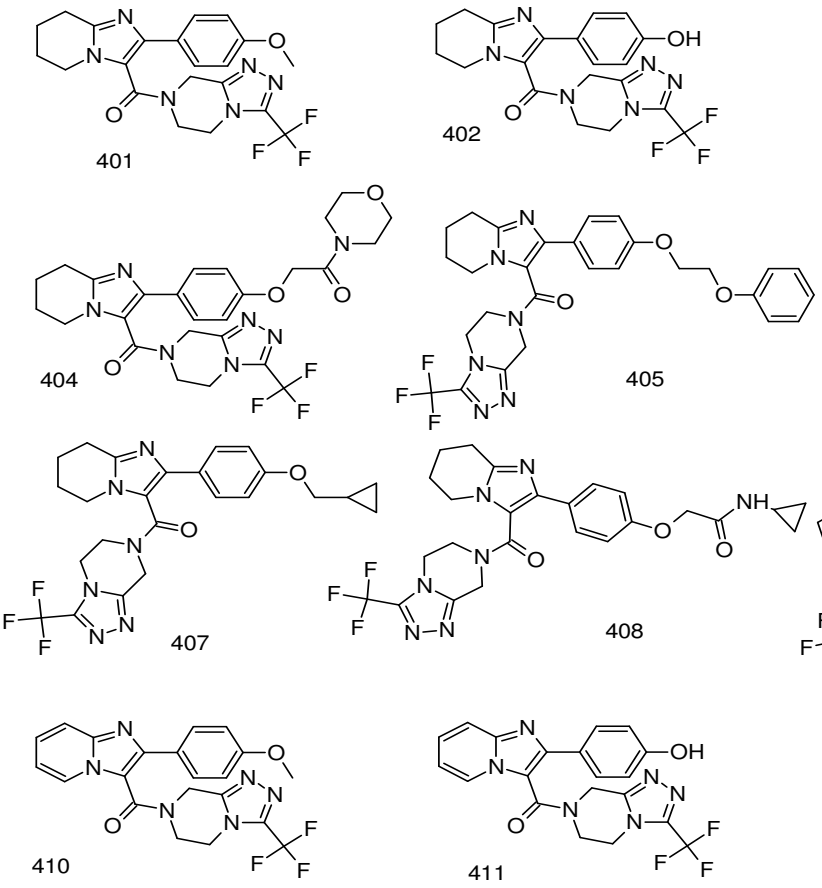

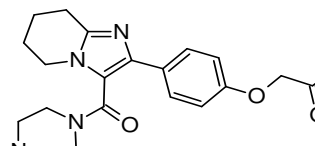<smiles>FC(F)(F)c1nnc2n1CCNC2</smiles>

408

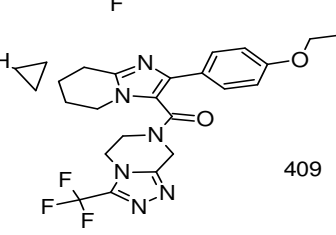<smiles>O=c1c2c(nc3n2CCCC3)c2ccc(OCCN3CCOCC3)cc2n1CCn1cnc(C(F)(F)F)n1</smiles><smiles>CC(C)CCOc1ccc(-c2nc3n(c2C(=O)N2CCn4c(nnc4C(F)(F)F)C2)CCCC3)cc1</smiles><smiles>[CH-]1CO1</smiles><smiles>O=C1Cn2nc(C(F)(F)F)nc2-c2nc(OCCN3CCOCC3)ccc2-c2nc3ccccn3c21</smiles><smiles>O=C(COc1ccc2c3nn(c2n1)CCN1C(=O)N(C(F)(F)F)CCN31)N1CCOCC1</smiles><smiles></smiles><smiles>CCCCCNc1ccc(F)cc1</smiles><smiles>O=C(c1nc2ccccn2c1-c1ccc(OCC2CC2)cc1)N1CCn2c(nnc2C(F)(F)F)C1</smiles>

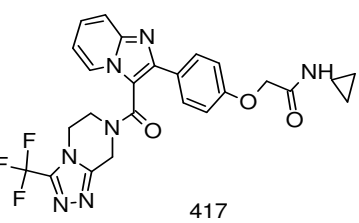<smiles>CCOc1ccc(-c2nc3ccccn3c2C(=O)N2CCn3c(nnc3C(F)(F)F)C2)cc1</smiles>

Figure 1. Compounds (401-418)

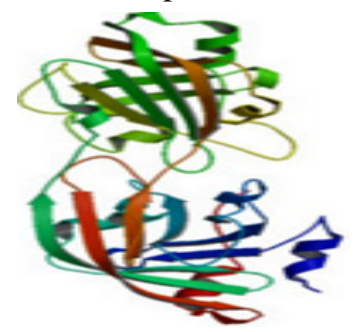

Figure 2. Sortase 
After the collecting PDB file of sortase from PDB databank, the possible binding sites of sortase was searched based on the structural comparison of template and the model build and also with CASTp server and was shown in Figure 3.

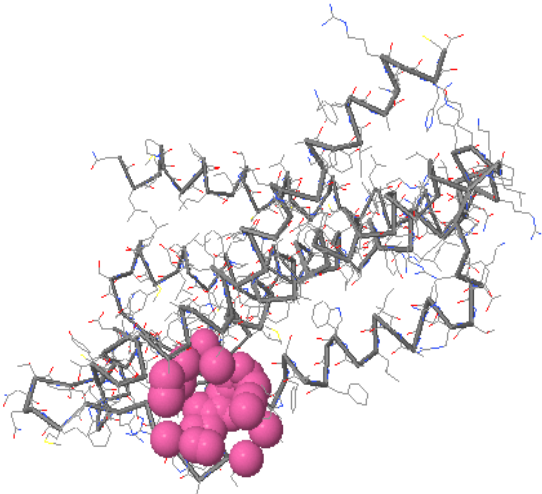

Figure 3. Active site of Sortase

It was found that secondary structures are highly conserved and the residues, TYR 98, LEU 147, TRP 148, VAL 149, ILE 150, PRO 151, ILE 152, TRP 155, PHE 180, MET 183, THR 184, ILE 187.

\section{Molecular docking study}

Docking studies were performed to gain insight into the binding conformation of pharmacophore models derived from structural manipulations onto derivatives. A library of lead molecules were constructed and screened for satisfying the minimal chemical criteria for further analysis using Molinspiration. The molecules were selected based on the criteria of satisfying Lipinski's Rule-of-Five with zero violations for docking onto Sortase models (Figure 4).

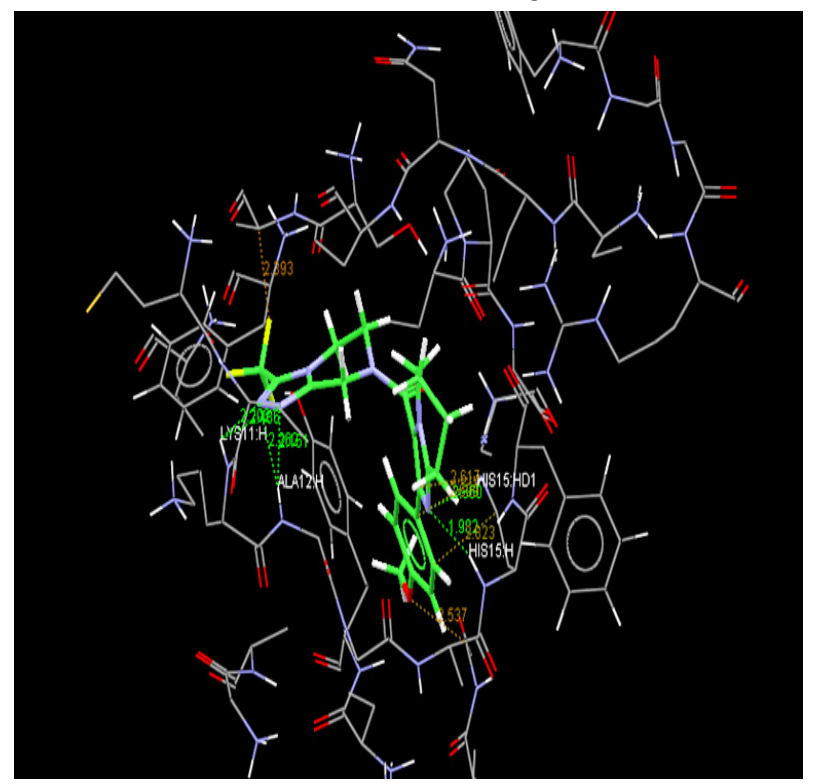

Figure 4a. Docking of 401 


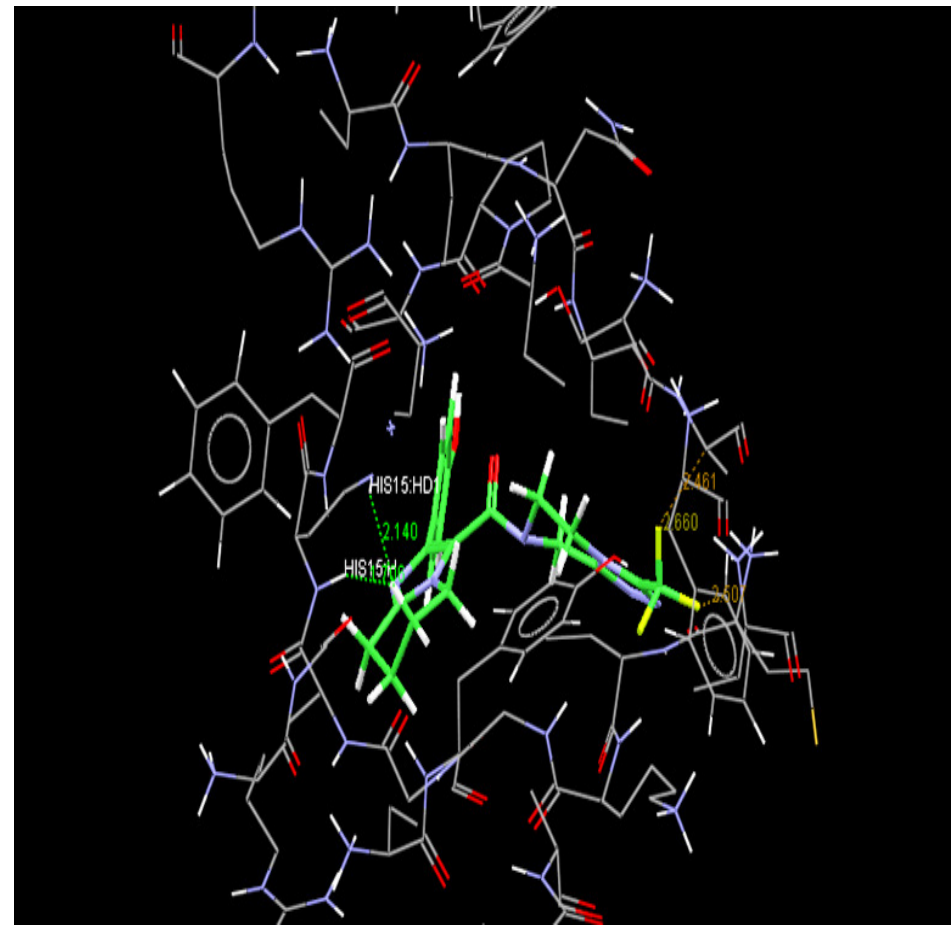

Figure 4b. Docking of 402

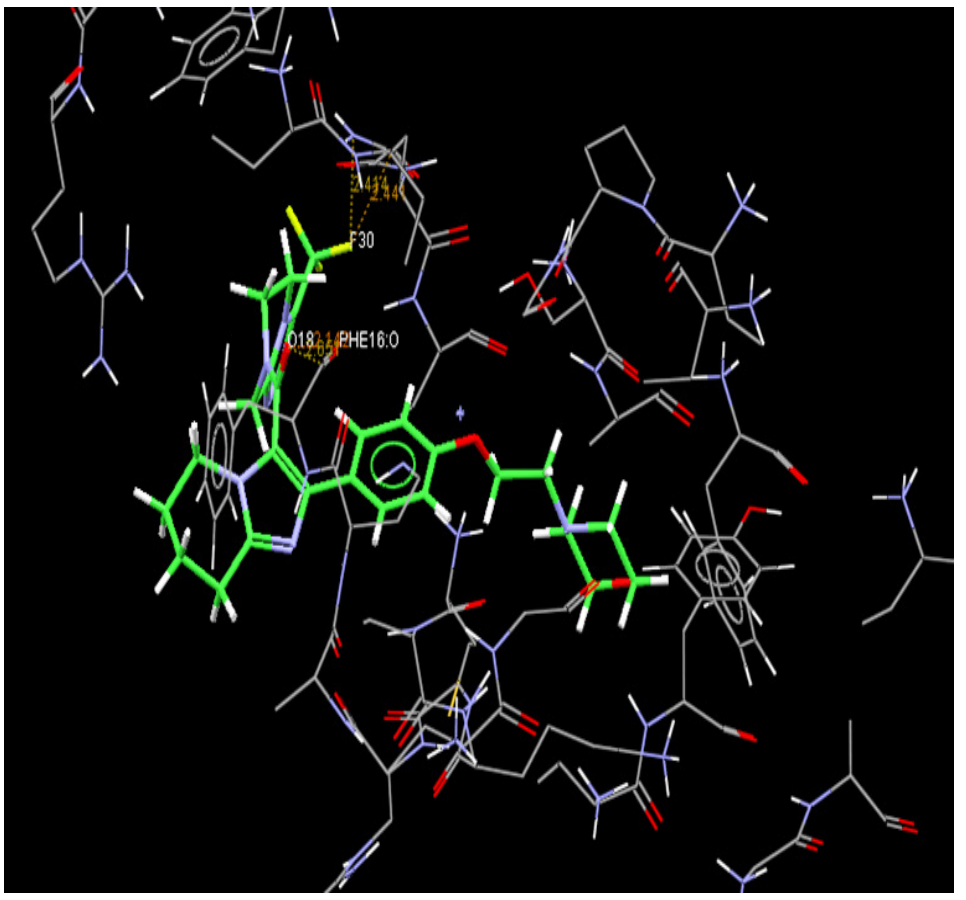

Figure 4c. Docking of 403 


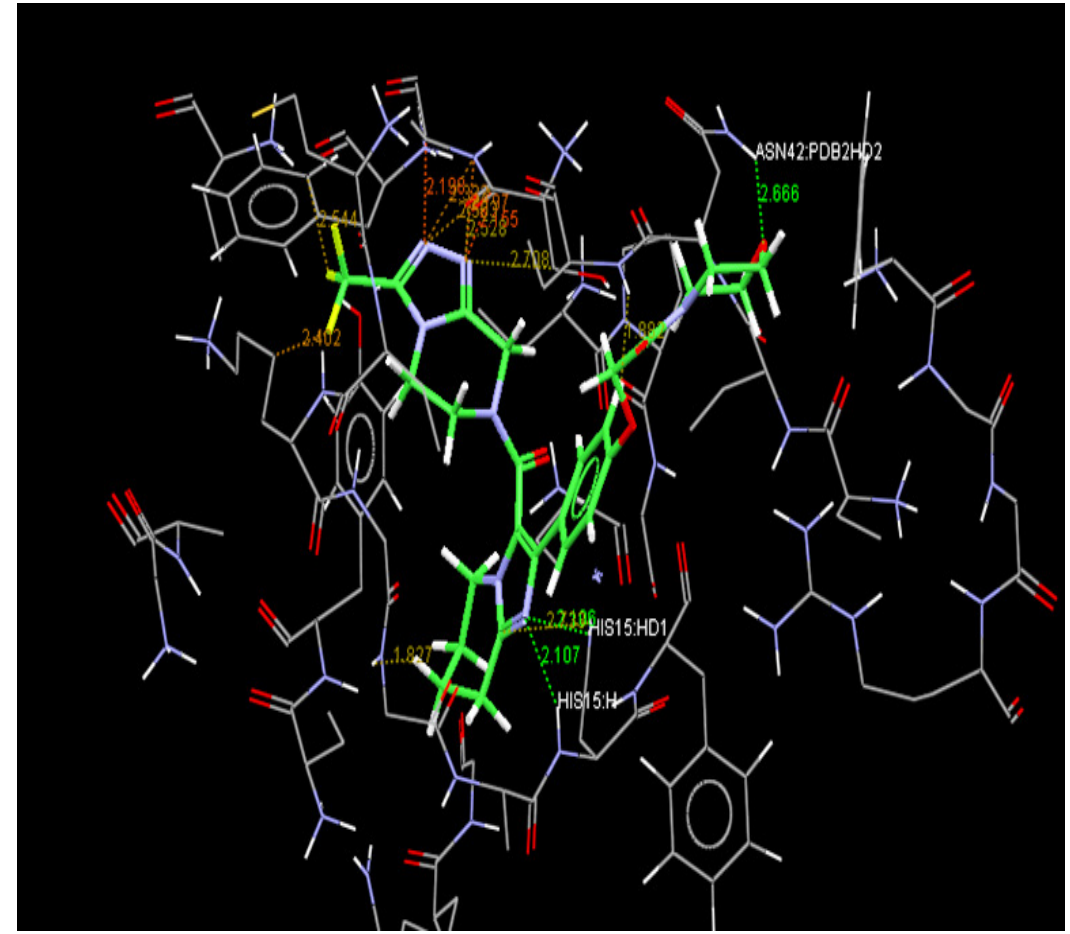

Figure 4d. Docking of 404

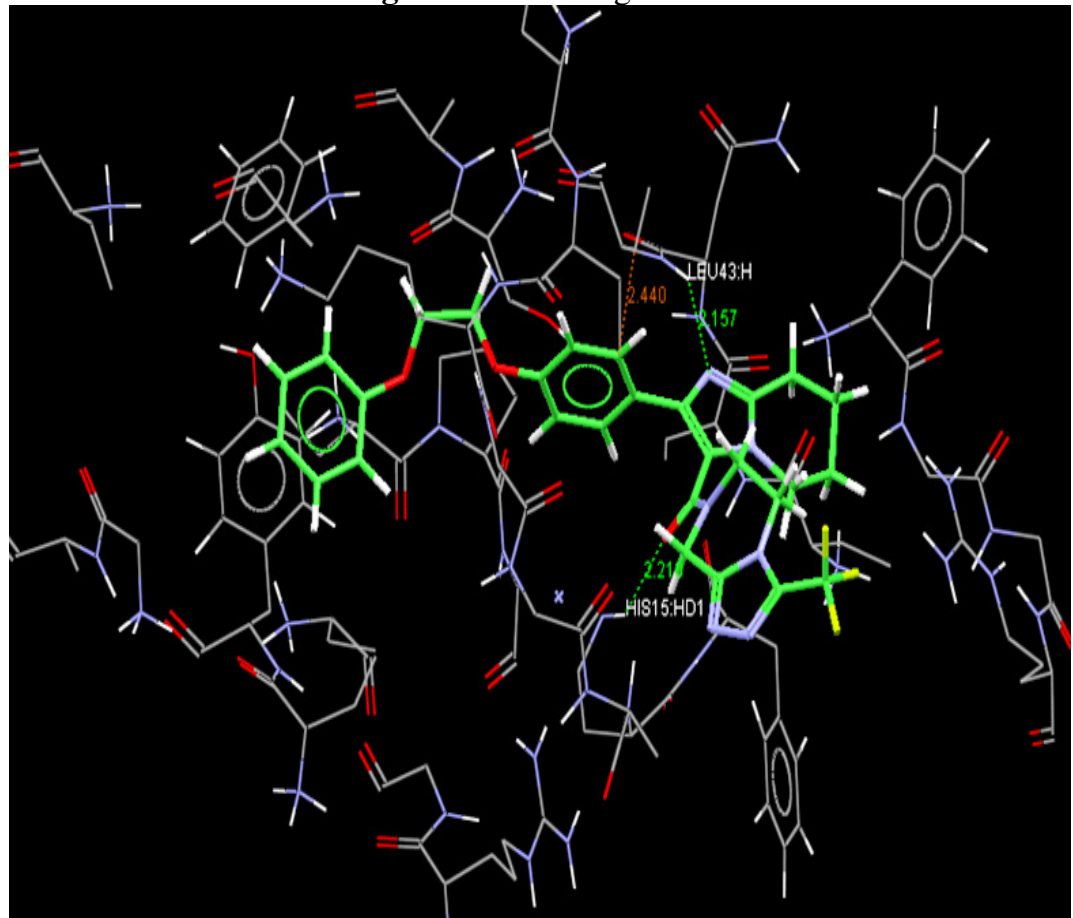

Figure 4e. Docking of 405 


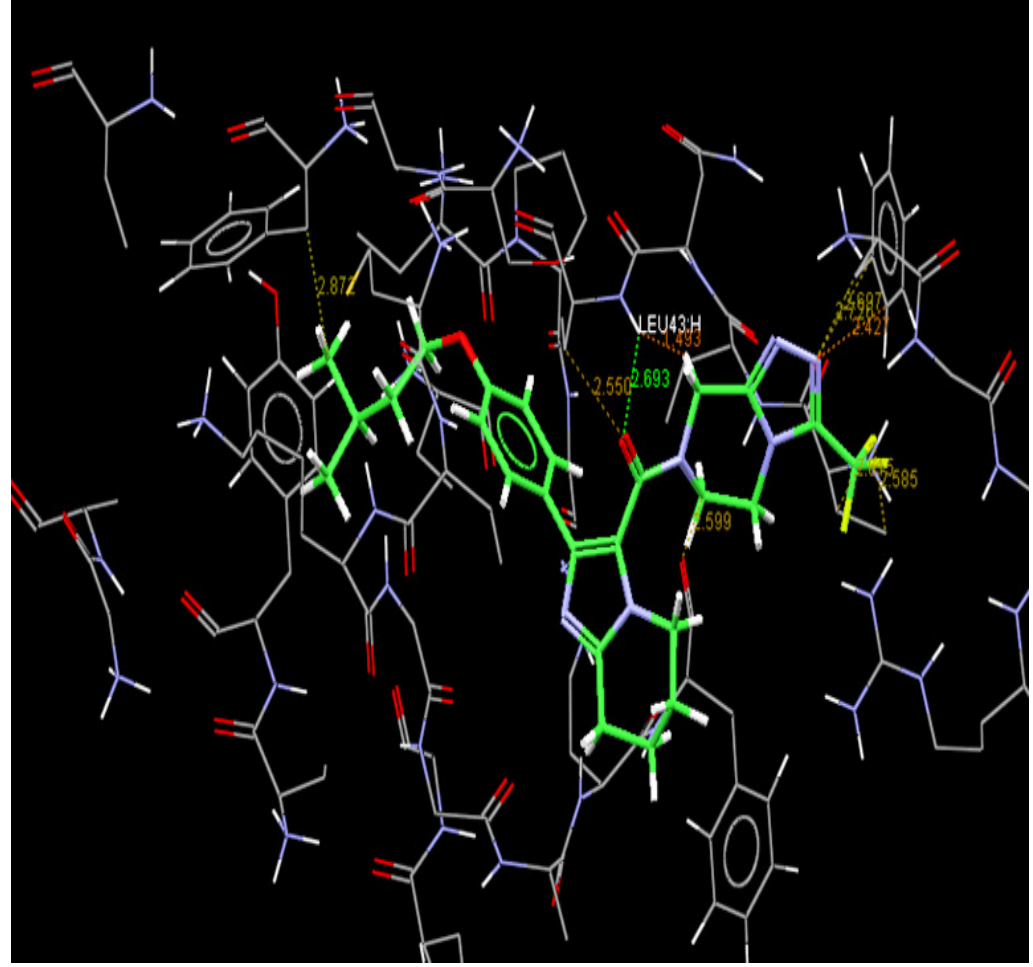

Figure 4f. Docking of 406

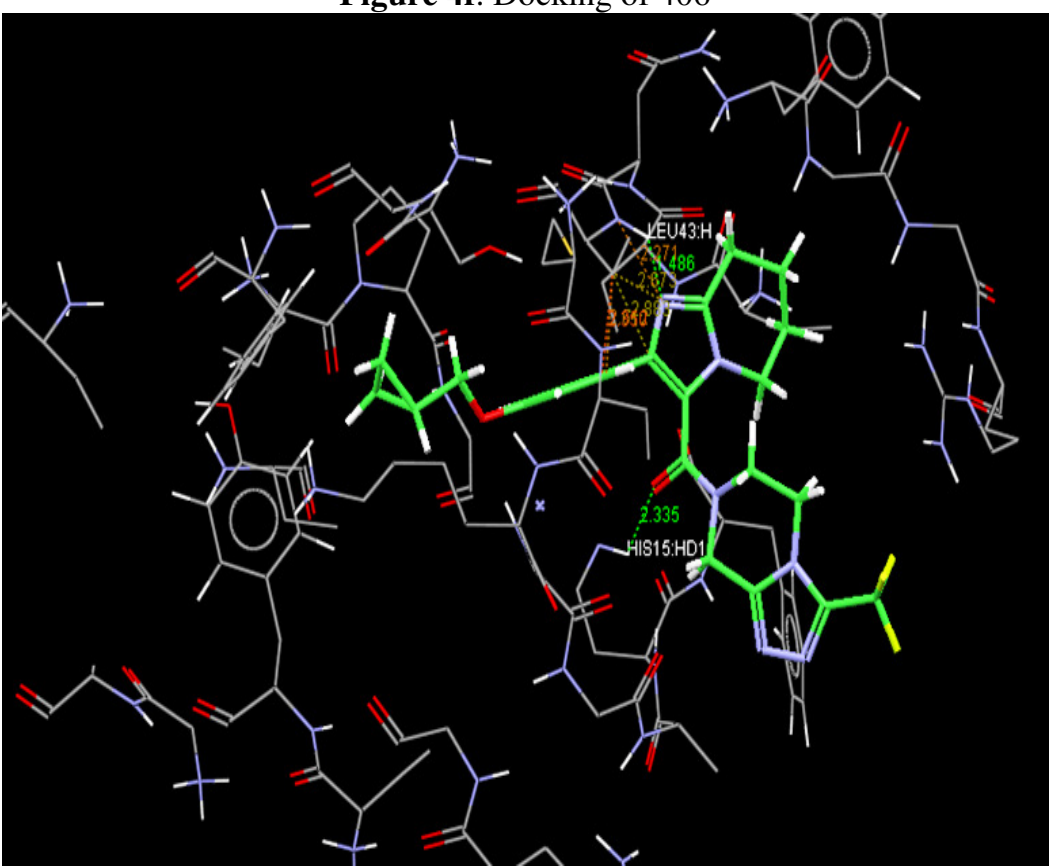

Figure 4g. Docking of 407 


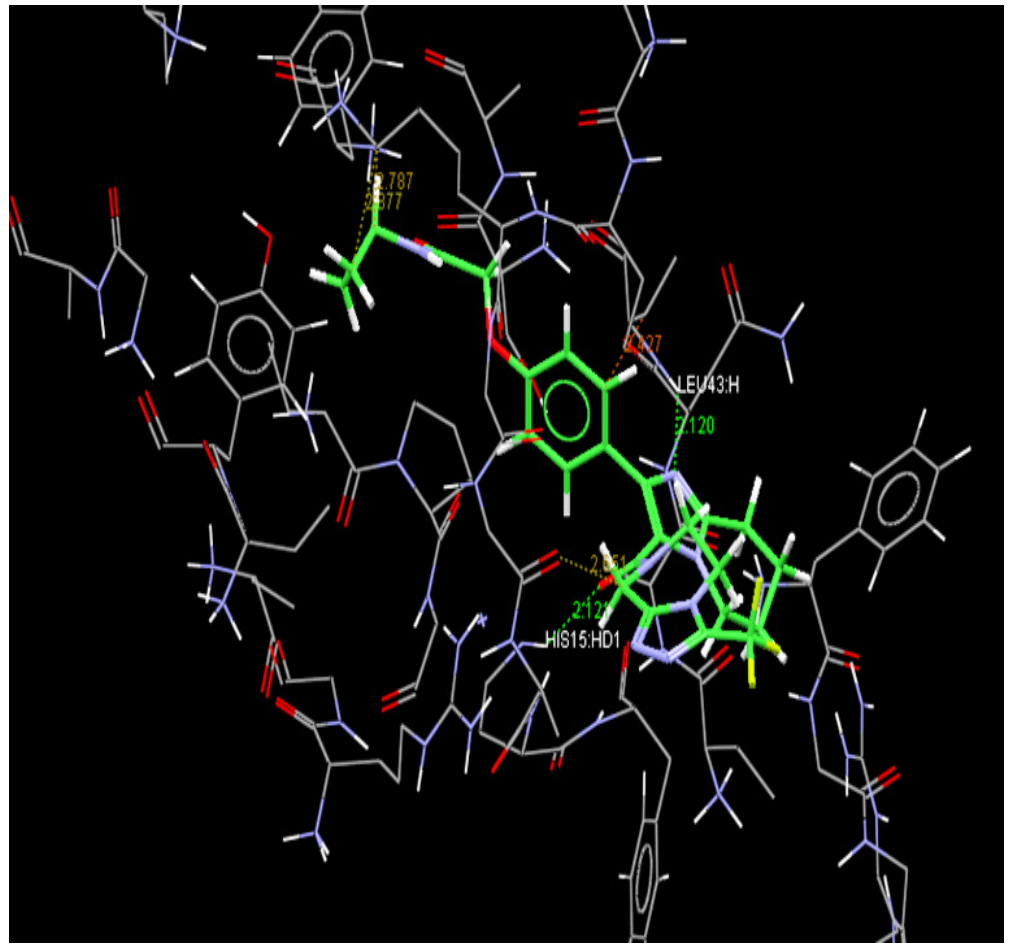

Figure 4h. Docking of 408

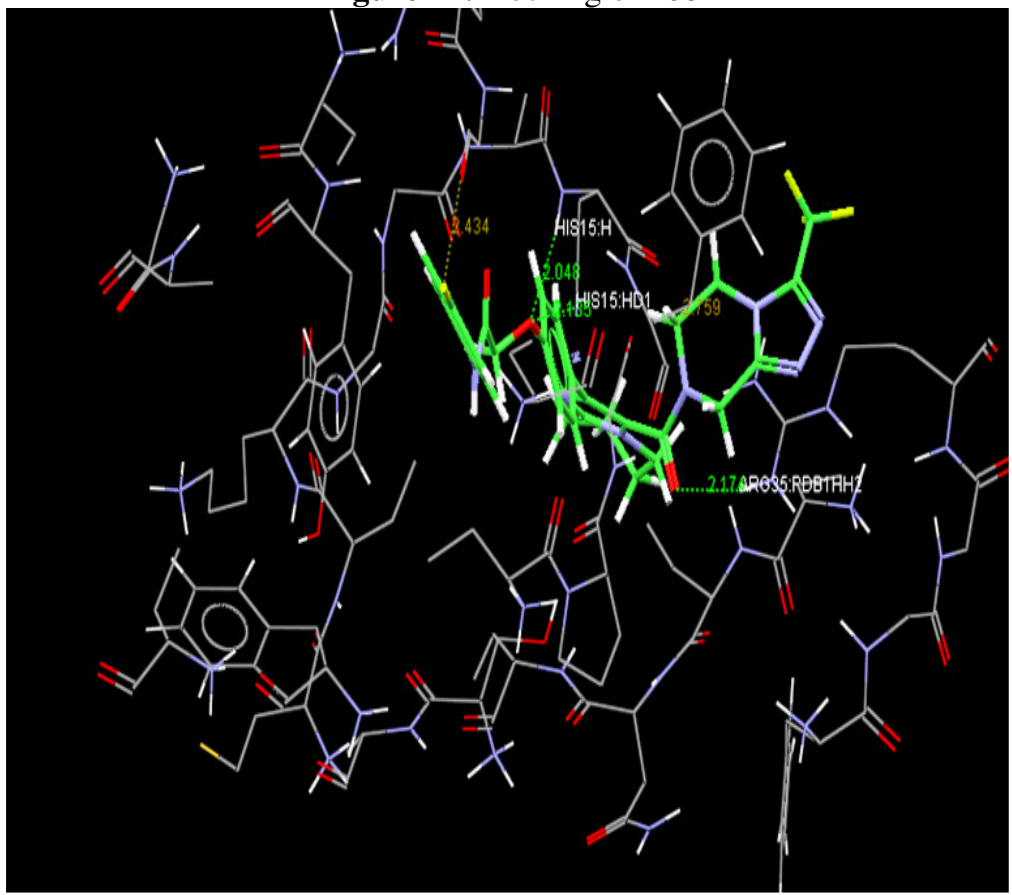

Figure 4i. Docking of 409 


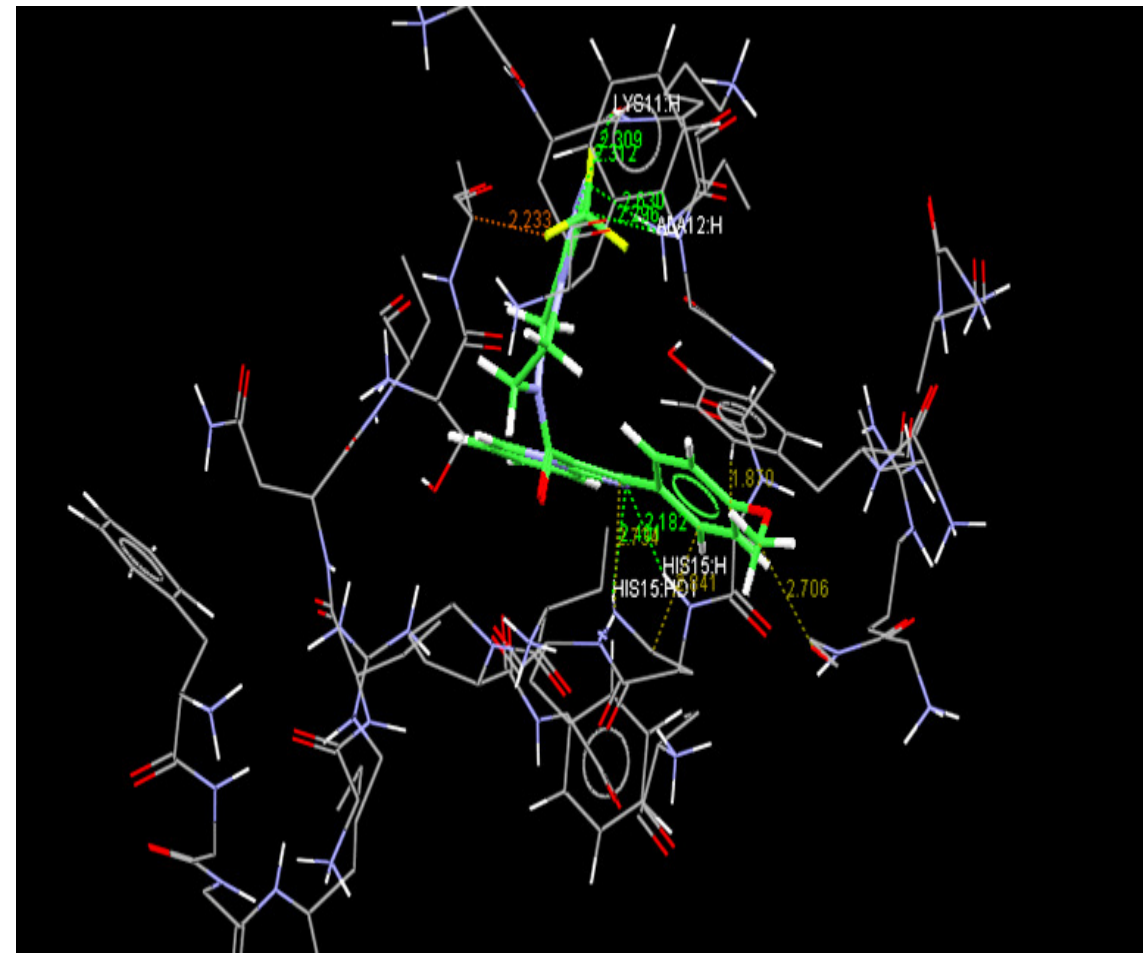

Figure 4j. Docking of 410

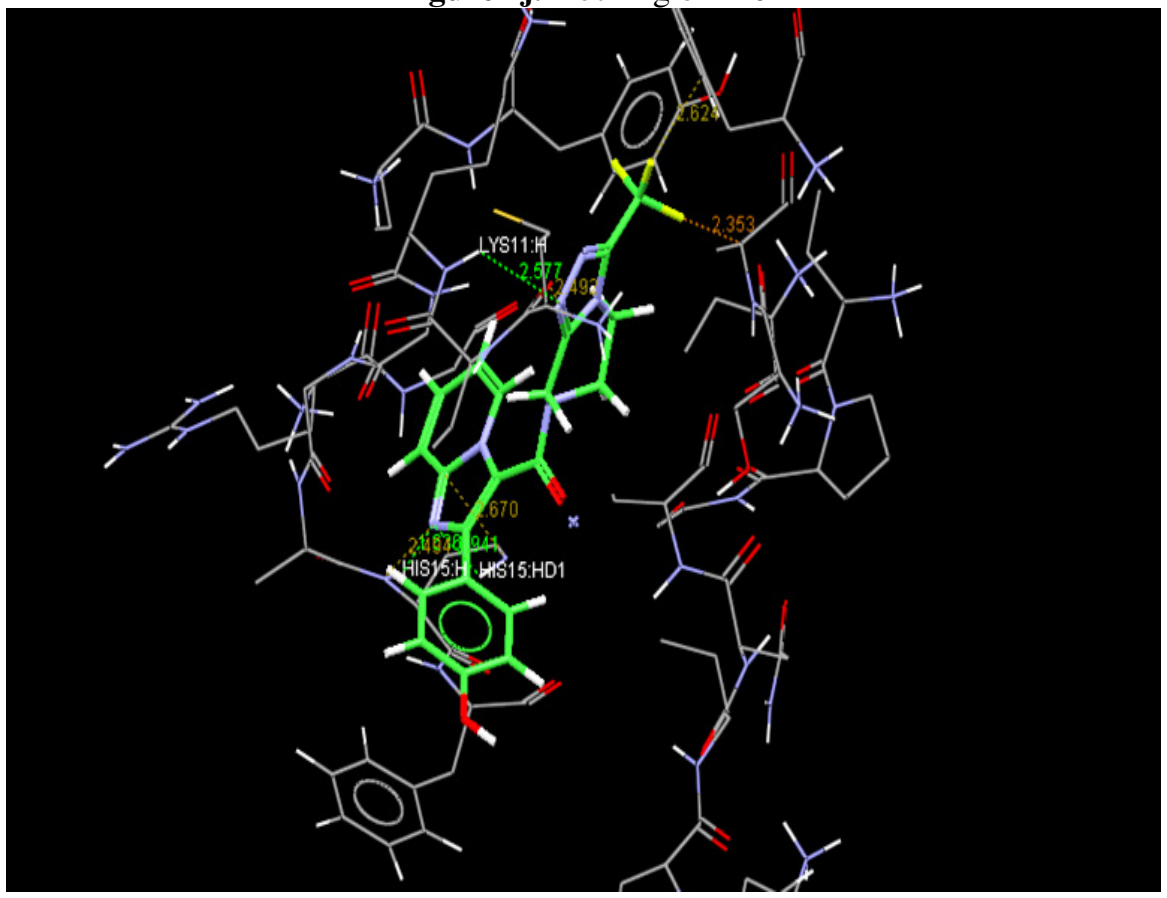

Figure 4k. Docking of 411 


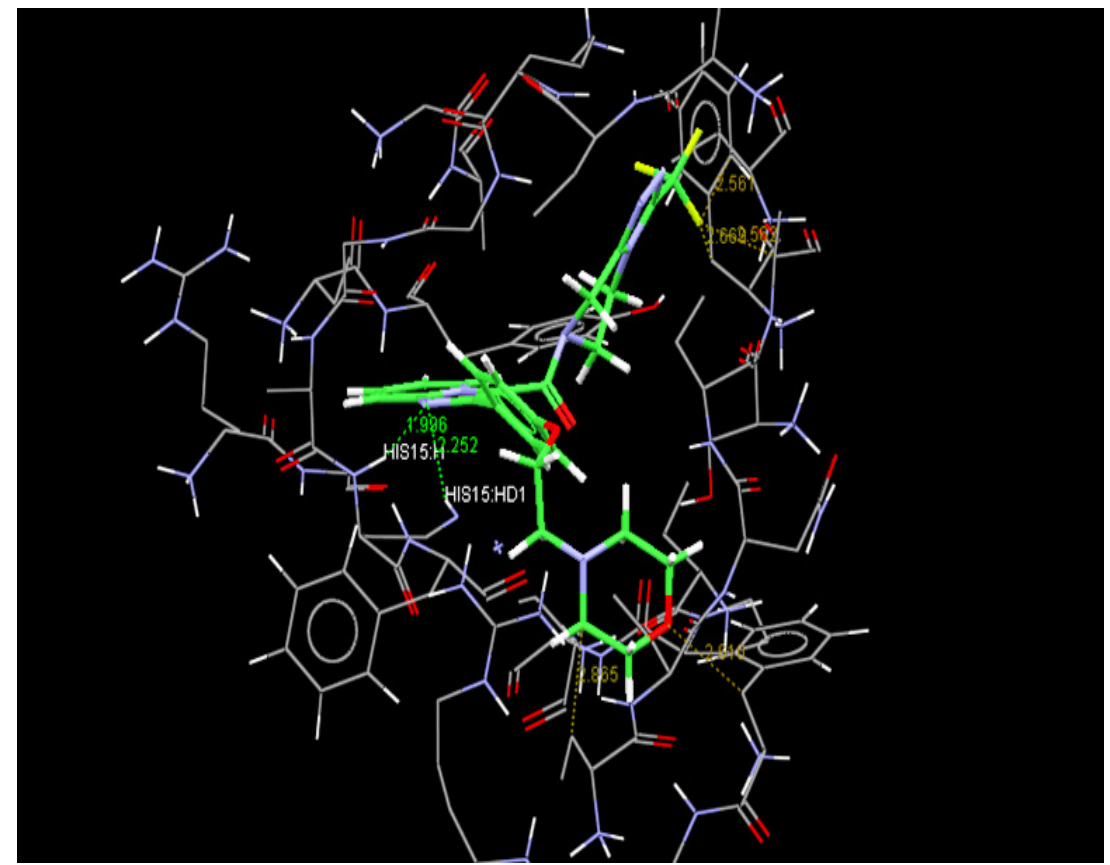

Figure 4l. Docking of 412

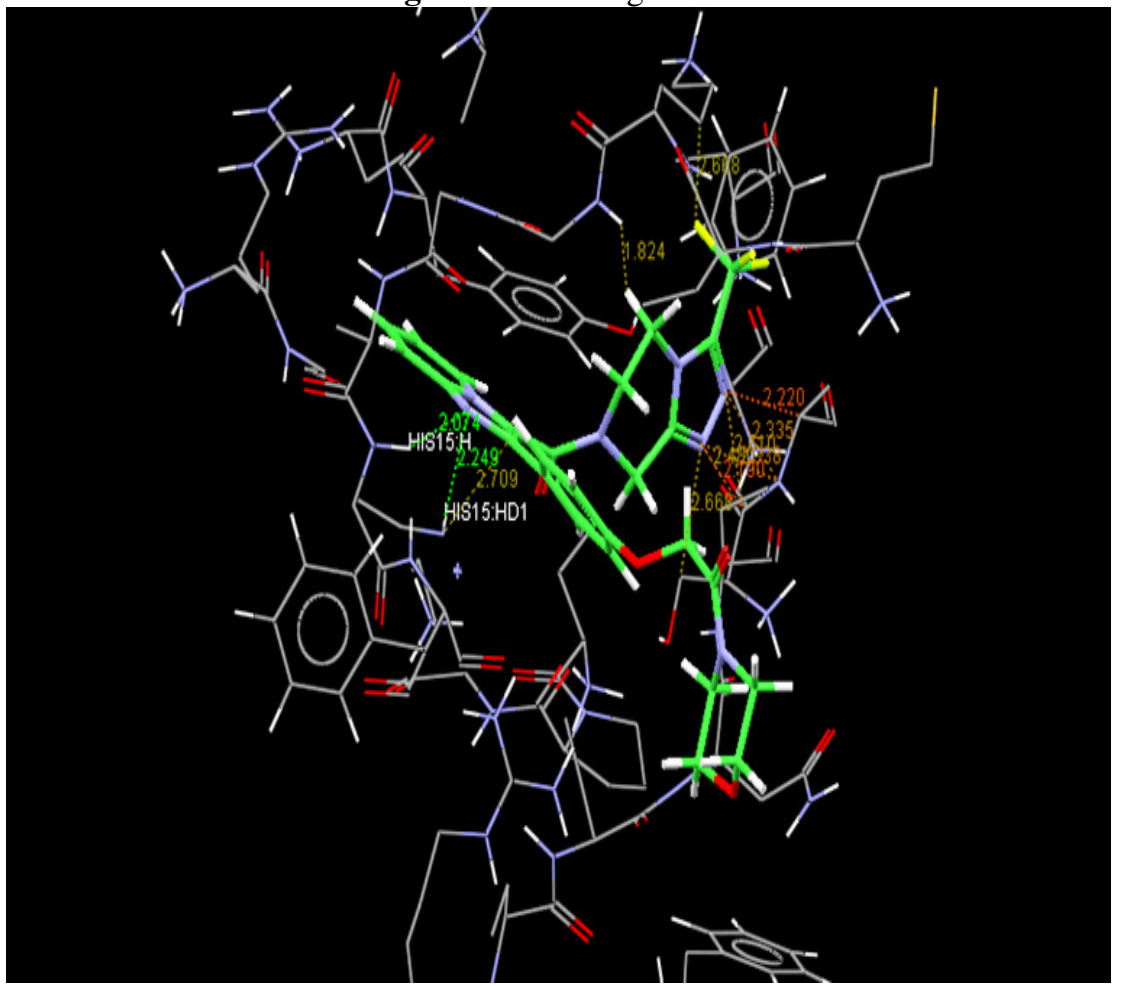

Figure 4m. Docking of 413 


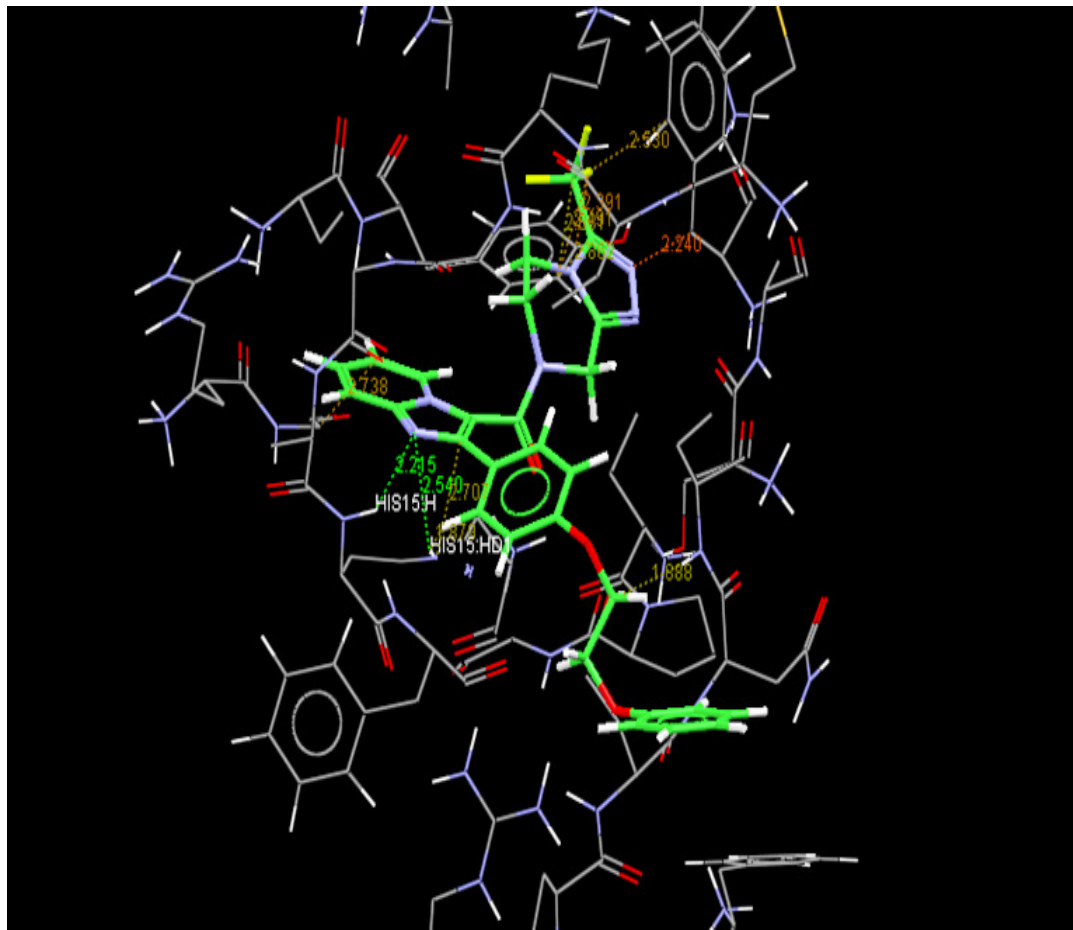

Figure 4n. Docking of 414

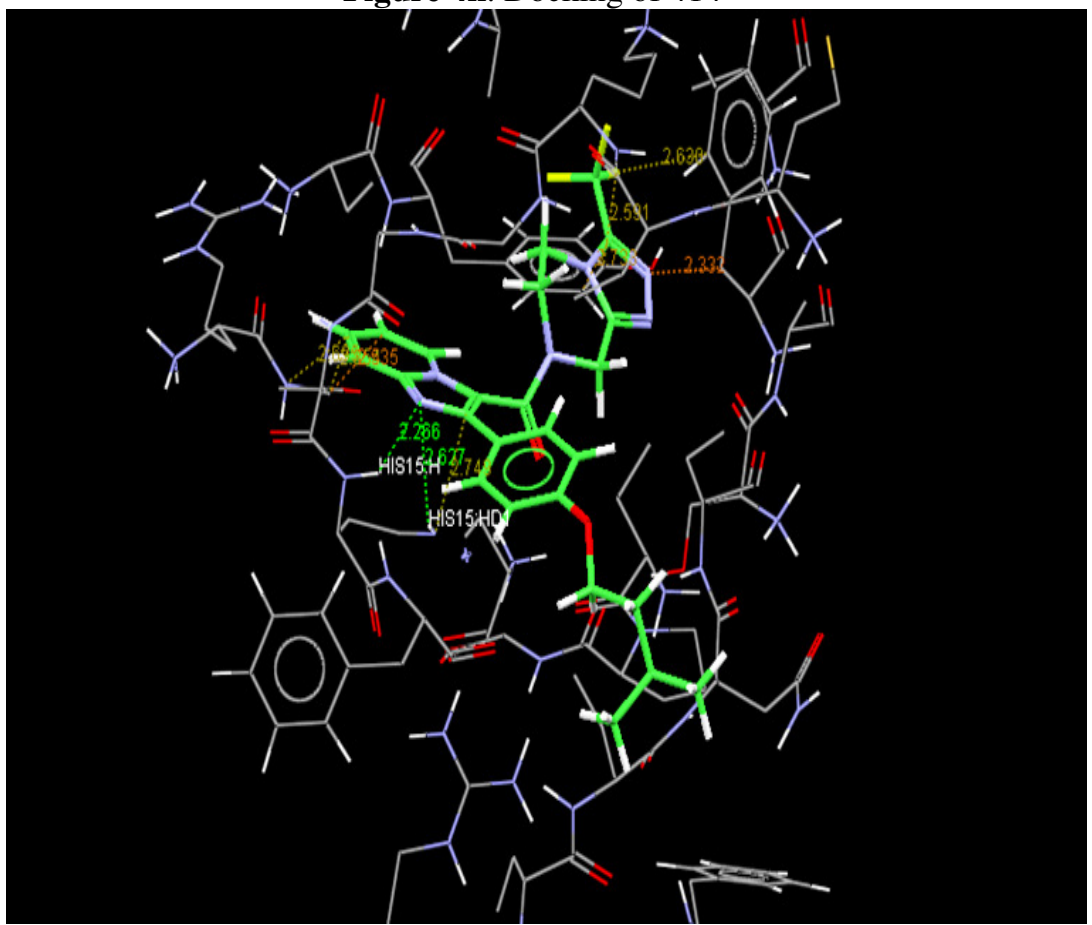

Figure 4o. Docking of 415 


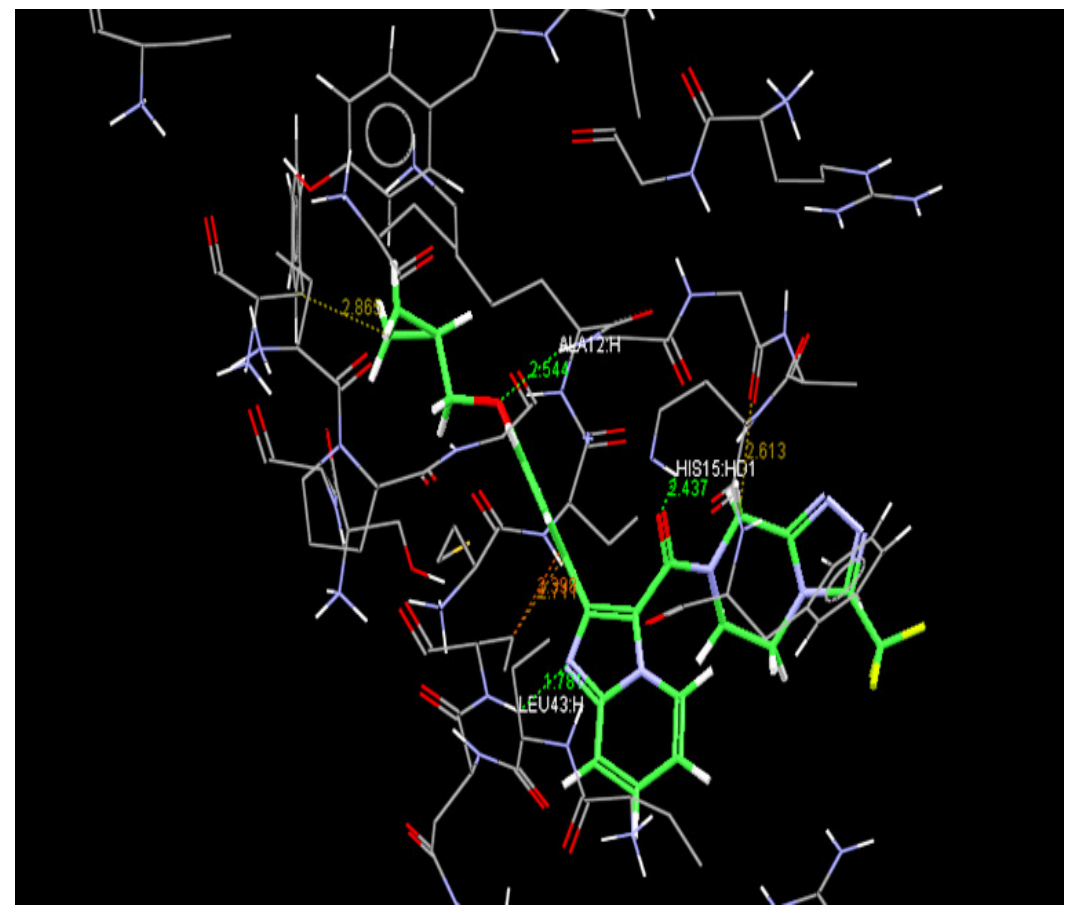

Figure 4p. Docking of 416

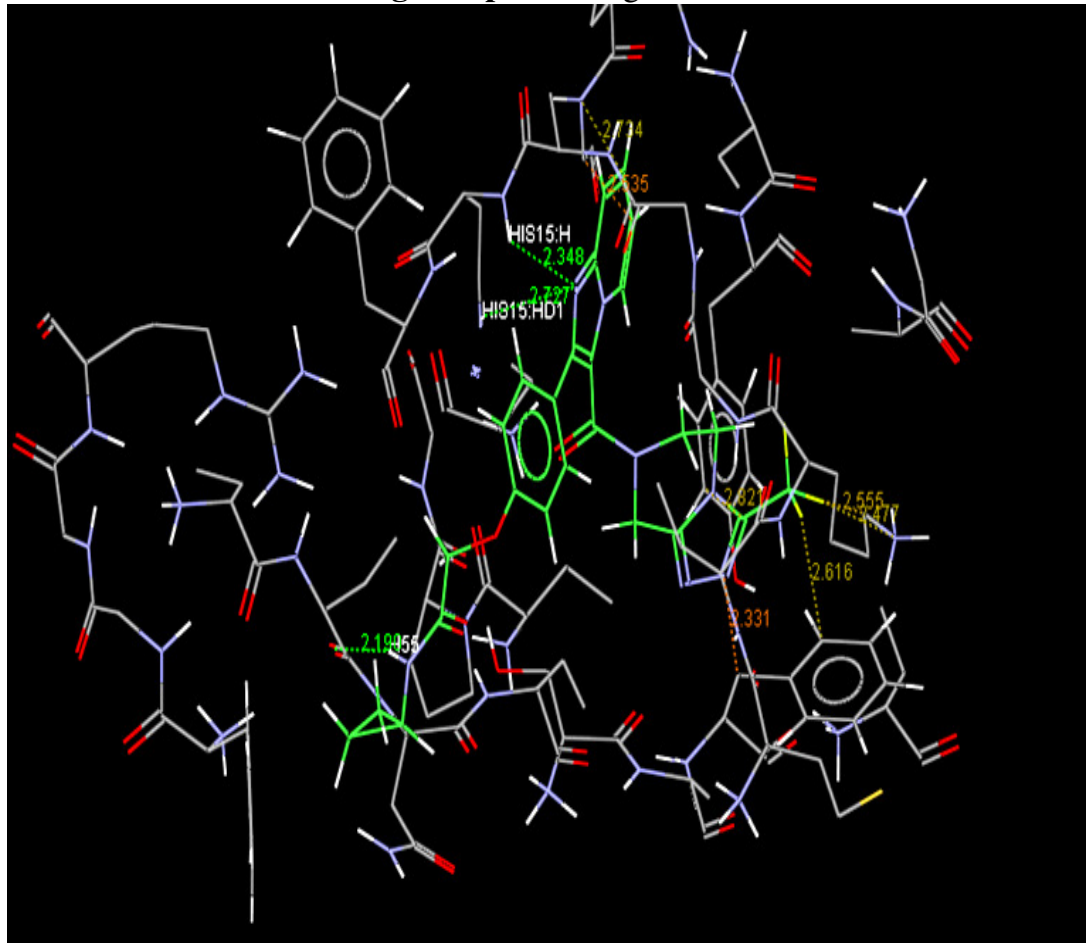

Figure 4q. Docking of 417 


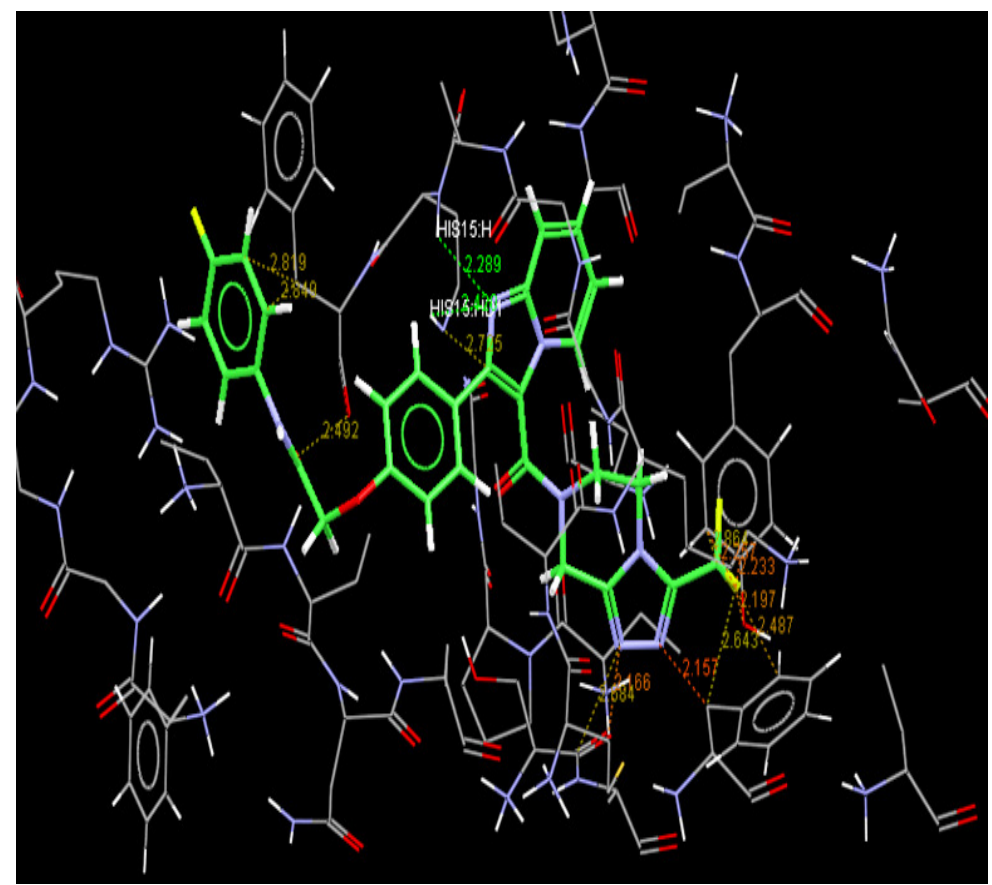

Figure 4r. Docking of 418

Figure 4. Docking studies of compounds (401-418)

All docking calculations were carried out using open eye and the files generated were analyzed for their binding conformations. Analysis was based on free energy of binding; Lowest docked energy and calculated RMSD values (Table 1).

Table 1. Fitness score

\begin{tabular}{cccccc}
\hline Fitness & S(hb_ext) & S(vdw_ext) & S(hb_int) & S(int) & Ligand name \\
\hline 58.20 & 1.23 & 49.03 & 0.00 & -10.45 & 401 \\
53.28 & 0.17 & 43.08 & 0.00 & -6.12 & 402 \\
50.46 & 0.00 & 45.43 & 0.00 & -12.00 & 403 \\
40.80 & 0.11 & 34.95 & 0.00 & -7.37 & 404 \\
54.33 & 0.62 & 47.30 & 0.00 & -11.33 & 405 \\
48.35 & 0.00 & 45.29 & 0.00 & -13.93 & 406 \\
42.25 & 0.35 & 39.76 & 0.00 & -12.76 & 407 \\
46.78 & 0.69 & 40.19 & 0.00 & -9.18 & 408 \\
55.55 & 3.40 & 45.68 & 0.00 & -10.66 & 409 \\
61.65 & 0.94 & 50.00 & 0.00 & -8.04 & 410 \\
53.40 & 0.25 & 44.19 & 0.00 & -7.62 & 411 \\
65.21 & 0.02 & 57.34 & 0.00 & -13.66 & 412 \\
45.06 & 0.04 & 36.44 & 0.00 & -5.08 & 413 \\
58.66 & 0.00 & 48.84 & 0.00 & -8.50 & 414 \\
56.81 & 0.00 & 48.80 & 0.00 & -10.29 & 415 \\
45.25 & 0.31 & 43.12 & 0.00 & -14.35 & 416 \\
63.39 & 1.46 & 50.28 & 0.00 & -7.21 & 417 \\
61.58 & 0.00 & 51.56 & 0.00 & -9.32 & 418 \\
\hline
\end{tabular}


The total clusters of docking conformations, with the eighteen docked lead molecules showed negative binding energies. Among all docking conformations compound 12 gave the best predicted binding free energy of $65.21 \mathrm{kcal} / \mathrm{mol}$ to the Sortase.

\section{Docking Results}

To understand the interaction between Sortase and drugs (L), the complex was generated using FRED 2.1, which is based on Rigid Body Shape-Fitting and the binding 3D conformation complex of the best docked drugs. This figure shows that drugs located in the center of the active site, and is stabilized by hydrogen bonding. Hydrogen bonds play an important role for structure and function of biological molecules, especially for enzyme catalysis. Among the docked drugs, $12^{\text {th }}$ compound is tightly bound by to the active site. The bonding of other drugs with Sortase was seen from Figure 4. To determine the key residues that comprise the active site of the model, the interaction energies of the drugs with Sortase were calculated. Table 1 shows deep interaction energies for all drugs. From the Table 1 we can see that among the P-L complexes, compound 12 has a large favorable total energy of $65.21 \mathrm{kcal} \mathrm{mol}^{-1}$. From these results we can suggest that PHE 180, MET 183, THR 184, ILE 187 are important residues in Sortase and are the main contributors to the inhibitor interaction. GLY-25, VAL-217 and HSD-220 are not involved in the bonding with drugs. However, we think that PHE 180, MET 183 AND ILE 187 may be important residues because these form hydrogen bonds with other drugs. From the alignment result, we can see that these four residues are highly conserved and these are important for enzyme catalysis.

\section{Conclusion}

Sortase is an important protein involves in the membrane formation and shape determination in staphylococcus aureus. In this work, we have collected 3D model of Sortase (PDB ID: 4O8L) domain, using the PDB method and obtained a refined model after energy minimization. The final refined model was further assessed by PROCHECK program, and the results show that this model is reliable. The stable structure is further used for docking with the ligand molecules. As is well known, hydrogen bonds play important role for the structure and function of biological molecules. In this study it was found that, TYR 98, LEU 147, TRP 148, VAL 149, ILE 150, PRO 151, ILE 152, TRP 155, PHE 180, MET 183, THR 184, ILE 187 are important for strong hydrogen bonding interaction with the inhibitors. To the best of our knowledge PHE 180, MET 183 AND ILE 187 are conserved in this domain and may be important for structural integrity or maintaining the hydrophobicity of the inhibitor-binding pocket. The molecule $12^{\text {th }}$ showed best docking results with target protein. The possible experimental values were identified from the docking results.

\section{References}

1. Ton-That H, Marraffini L A and Schneewind O, Biochim Biophys Acta, 2004, 1694(1-3), 269-278; DOI:10.1016/j.bbamcr.2004.04.014

2. Mazmanian S K, Liu G, Jensen E R, Lenoy E and Schneewind O, Proc Natl Acad Sci USA, 2000, 97(10), 5510-5515; DOI:10.1073/pnas.080520697

3. Mazmanian S K, Liu G, Ton-That H and Schneewind O, Science, 1999, 285, 760-763.

4. Marraffini L A, Ton-That H, Zong Y, Narayana S V L and Schneewind O, J Biol Chem., 2004, 279, 37763-37770; DOI:10.1074/jbc.M405282200

5. Schneewind O, Fowler A and Faull K F, Science, 1995, 268, 103-106.

6. Mazmanian S K, Ton-That H, Su K and Schneewind O, Proc Natl Acad Sci USA, 2002, 99, 2293-2298. 